\title{
La civilité électorale : vote et forclusion de la violence en France (Partie 1)
}

Olivier Ihl

\section{(2) OpenEdition \\ Journals}

Édition électronique

URL : http://journals.openedition.org/conflits/408

DOI : $10.4000 /$ conflits.408

ISSN : $1777-5345$

Éditeur :

CCLS - Centre d'études sur les conflits lilberté et sécurité, L'Harmattan

Édition imprimée

Date de publication : 15 mai 1993

ISSN : 1157-996X

\section{Référence électronique}

Olivier Ihl, «La civilité électorale : vote et forclusion de la violence en France (Partie 1) », Cultures \&

Conflits [En ligne], 09-10 I printemps-été 1993, mis en ligne le 27 janvier 2003, consulté le 30 mars

2021. URL : http://journals.openedition.org/conflits/408; DOI : https://doi.org/10.4000/conflits.408

Ce document a été généré automatiquement le 30 mars 2021.

Creative Commons License 


\title{
La civilité électorale : vote et forclusion de la violence en France (Partie 1)
}

\author{
Olivier Ihl
}

1 Les incitations ne manquent pas qui poussent les acteurs sociaux à concevoir l'ordre politique moderne comme un idéal de rationalité et de fonctionnalité garant d'une véritable pacification des esprits et des corps. En caractérisant l'Etat à travers sa capacité à revendiquer "le monopole de la contrainte physique légitime" ${ }^{1}$, Max Weber établit une affinité très forte entre la forclusion de la violence privée et l'avènement d'un mode de domination rationnelle-légale. Parce qu'"il n'existe de nos jours de violence "légitime" que dans la mesure où l'ordre étatique la permet ou la prescrit" ${ }^{2}$, les éruptions périodiques de violence ou de protestation qui affectent la vie politique française paraissent difficile à comprendre. Nuançant l'hypothèse centrale de la Dynamique de l'Occident proposée par Norbert Elias, ces formes rebelles d'action politique rappellent la fragilité des mécanismes institutionnels et culturels censés pacifier l'espace public. Leur présence invite par là même la science politique à s'intéresser aux différentes modalités de gestion de la violence. Quelles sont les raisons qui obligent les citoyens à respecter l'ordre pacifié de l'Etat? A peine formulée, une telle question signale la nécessité de déplacer la réflexion de son cours habituel. Il s'agit moins de comprendre la progressive monopolisation de la violence physique par l'Etat que d'analyser l'entreprise d'acculturation civique qui l'accompagne nécessairement. C'est grâce à cette dernière que les relations sociales et politiques se voient sommées de perdre leurs attributs conflictuels. Le rejet de la violence en tant que mode d'action politique impose notamment de créer des espaces "neutralisants" susceptibles de dissiper la menace du contact social. La mise en oeuvre de l'activité électorale occupe, à cet égard, une place déterminante. Le vote tend à opposer à la rhétorique vindicative de la force une autre scénographie: celle d'une adhésion silencieuse par laquelle s'établit rituellement mais pacifiquement le mécanisme politique de la délégation. C'est dire si les vertus civiques qui forment l'ossature de la construction normative du 
suffrage universel sont inséparables d'un projet plus général de forclusion de la contrainte physique ${ }^{3}$.

Quel peut être l'intérêt de mettre en parallèle l'expérience électorale avec le phénomène de la violence politique ? Il est d'abord de libérer certains des problèmes théoriques que soulève l'inscription d'un tel geste dans la trame quotidienne des relations sociales. La pratique du scrutin ne reste pas sans effet sur ses utilisateurs. Elle heurte, bouleverse, transforme leurs conduites car ses modalités particulières pèsent directement sur les usages qui viennent périodiquement l'investir. Il est ensuite de montrer que la pratique du vote s'apparente non seulement à une technique d'enregistrement des "opinions" mais aussi à un outil d'apprentissage et d'acculturation. Sa généralisation à partir de la fin du XIXe siècle s'établit sur un double mouvement : d'une part, l'émancipation des formes traditionnelles de rapport à soi qui favorisaient l'explosion des violences tantôt communautaires tantôt révolutionnaires, d'autre part, la diffusion d'un système de représentations célébrant la primauté de la conscience individuelle comme principe légitime de choix politique.

3 A partir de ce cadre théorique, on voudrait examiner les principales modalités d'institutionnalisation d'un espace pacifié du vote, notamment celles qui se rapportent au travail juridique de codification. Il conviendra également de dire un mot des formes de représentation qui forgent et pérennisent un pareil ordonnancement électoral tout en restant bien sûr attentif aux multiples résistances qu'a pu rencontrer historiquement l'application de ces règles de civilité électorale.

\section{L'ESPACE DU VOTE COMME ESPACE DE CIVILITE}

4 Au départ, un constat : la dimension pacificatrice de l'activité électorale est un thème largement retenu par les principaux illustrateurs du suffrage universel. Le plus célèbre d'entre eux, Honoré Daumier ne représente-t-il pas, en 1869, un ouvrier républicain plein de fougue à l'idée de voter. Brandissant son bulletin de vote, cet électeur clame tout haut: "V'là ma cartouche" ${ }^{4}$. Le message est clair : le bulletin de vote est présumé posséder la même valeur et la même force que la cartouche du révolutionnaire de 1848 . Représentation imagée de l'acte du vote, le dessin définit l'élection comme le seul mode légitime de dévolution du pouvoir. A considérer de plus près cette figure topique du discours républicain ${ }^{5}$, on constate qu'elle tend - en pratique - à accréditer l'idée d'une frontière: celle qui doit séparer l'espace du vote de la vie extérieure. Elle vise à instituer, en somme, comme un dedans et un dehors grâce auxquels l'acte de vote sera mis à l'abri des pressions et des violences sociales ou politiques. Prolongeant ces attendus normatifs, la législation électorale se montre particulièrement sourcilleuse. La violence n'est-elle pas l'expression la plus antagonique du droit de suffrage ? N'est-elle pas l'affirmation en acte de la volonté de certains d'infléchir et donc de corrompre le destin d'autrui ? N'est-elle pas, plus fondamentalement, un mode de domination tenu pour archaïque ? Rétrospectivement, la mise en place de tels lieux semble aller de soi, et ce d'autant plus que le processus de neutralisation s'est étendu désormais à l'ensemble de l'espace public. Le résultat en est une difficulté à penser les enjeux du vote en dehors des considérants qui définissent l'éthique démocratique. D'où l'intérêt d'une lecture, même rapide, des dispositions juridiques mises en oeuvre tout au long du XIXè siècle pour opérer une telle partition.

Intérieur et extérieur

5 Nul espace n'est plus réglementé que l'espace public du vote. Régi par un droit spécifique : le droit électoral, les opérations du vote font l'objet d'une vigilance toute 
particulière de la part du législateur. Il est frappant de constater que durant la Révolution la question - pourtant essentielle - de la sincérité du suffrage (et donc des moyens et des dispositions qui ouvrent sa garantie) est en partie occultée par la nécessité d'établir le libre accès de la salle de vote. La priorité est d'éviter que des gestes de violence n'empêchent l'électeur d'accomplir ce qui va devenir "son" devoir civique. Même avant la proclamation du suffrage universel, la législation électorale punit très sévèrement les actes de brutalité matérielle dirigés contre l'assemblée électorale. Signalons les articles 616 et suivants du Code du 3 brumaire an IV qui établissent que "tous complots ou attentats pour empêcher la réunion (...) d'une assemblée électorale, seront punis de la peine de la gêne pendant 15 ans" (art. 616) et que "quiconque sera convaincu d'avoir par force ou violence écarté ou chassé d'une assemblée primaire un citoyen ayant droit d'y voter, sera puni de la peine de la dégradation civique" (art. 617). L'article 618 stipule quant à lui que "si des troupes investissent le lieu des séances d'une assemblée primaire ou électorale, ou pénètrent dans son enceinte sans l'autorisation ou la réquisition de son président, les membres du Directoire Exécutif, ou le ministre, ou le commandant qui en auront donné l'ordre, et les officiers qui l'auront fait exécuter, seront punis de la peine de la gêne pendant 15 ans"'.

6 La sévérité des sanctions envisagées s'ancre dans les préoccupations d'une époque : il s'agit d'assurer la libre convocation des assemblées électorales. Mais aussi de protéger ces dernières ressurgissements d'une foule dont on craint encore la mobilisation réactive. Autrement dit, l'objectif est de permettre aux électeurs de s'accoutumer à une pratique encore fragile car faiblement institutionnalisée. Expression des valeurs morales d'une société en proie au vertige d'un retour de la Révolution, la règle juridique n'a qu'une seul finalité : disqualifier politiquement l'usage de la violence contre et dans les assemblées électorales. Pour cela, est établi une sorte de cordon protecteur : la prohibition de l'usage des armes dans la salle de vote. La seule exception prévue concerne une réquisition conduite par le président du bureau de vote. Dans ce cas, c'est la violence légitime qui est convoquée afin de rétablir l'ordre menacé et favoriser le déroulement normal du scrutin. En dehors de cette configuration exceptionnelle, les forces armées se voient exclues d'un espace de vote en cours de neutralisation. Ce caractère de neutralité procède, pour l'essentiel, de la mise en forme spatiale du geste lui-même ${ }^{7}$ : le vote s'inscrit désormais dans un espace réglementé qui protège l'électeur des violences du monde extérieur. Il est délimité par une zone où s'exercent des normes topographiques appropriées. Au sein des frontières matérielles que pose le droit électoral, domine l'impératif moral du contrôle de soi, c'est-à-dire l'exigence d'une maitrise du corps et des passions. Le schéma est donc simple: l'électeur est censé apprendre de nouvelles règles de conduite afin de se conformer aux propriétés de l'espace ainsi délimité. En ce sens, l'acte de vote renvoie à un processus plus large. Il participe de l'avènement d'une véritable civilité électorale. N'implique-t-il pas déjà un abaissement progressif du seuil de sensibilité à la violence, analogue à celui mis en évidence par Norbert Elias sur le terrain des manières de table, d'hygiène ou de lit $^{8}$ ?

7 L'entreprise juridique de pacification du bureau de vote se poursuivit tout au long $d u$ XIXème siècle. Le premier Code pénal, rédigé en 1810, reprend les principales dispositions imaginées pendant la Révolution. L'article 109 de ce code stigmatise tout particulièrement les "attroupements, voies de fait ou menaces" qui auront "empêché un ou plusieurs citoyens d'exercer leurs droits civiques". Fait significatif: pour 
répressive qu'elle soit, la législation de l'époque ne parle pas des formes non violentes d'incitation à l'abstention (achat d'un non-déplacement au bureau de vote, par exemple). Il est vrai qu'avec le suffrage restreint, c'est plus l'accès au bureau et le bon déroulement $\mathrm{du}$ scrutin qui inquiètent les juges que la corruption électorale proprement dite. La jurisprudence qui accompagne ces dispositions précise que la protection dont bénéficie l'acte du vote doit également être étendue au résultat du scrutin lui-même. De même que sont prohibées les violences faites à l'électeur, des mesures sont édictées pour empêcher l'altération de l'urne ou des bulletins de vote que cette dernière contient ${ }^{9}$.

8 L'instauration $\mathrm{du}$ suffrage universel rendit particulièrement nécessaire le renforcement des dispositions du Code pénal. Aussi la loi électorale du 15 mars 1849 at-elle réservé son titre VI à cette question. Bien qu'éphémères dans leur application, ces prescriptions juridiques survécurent à la loi de 1849. Elles furent reprises presque littéralement dans le titre IV du décret organique du 2 février 1852 qui allait organiser pour longtemps les opérations électorales en France. Au terme de ce décret fondateur, les armes sont définitivement proscrites de la salle de vote. L'article 37 dispose, en effet, que "l'entrée dans l'Assemblée électorale avec armes apparentes est interdite" : le contrevenant sera passible d'une amende de seize à cent francs. Au cas où les armes sont dissimulées ou cachées, l'amende s'accompagne d'une peine d'emprisonnement de quinze jours à trois semaines ${ }^{10}$. Sous la forme d'une interdiction pure et simple, la mesure s'applique non seulement aux particuliers mais aux agents de la force publique. L'arsenal répressif mis en place démontre à la fois l'importance qu'accorde le législateur à la pacification du lieu de vote et l'insuffisante institutionnalisation de la pratique électorale. Faute d'être un rite ancré dans les habitudes et les conventions ou une institution au sens où l'entend Mary Douglas ${ }^{11}$, l'acte de vote se voit encadré par une législation pénale très poussée. Cette attention révèle également la crainte qui domine alors les élites politiques. Celles-ci, à l'instar de Montalembert, redoutent que le suffrage universel ne soit pas "une digue suffisante pour arrêter les nouvelles irruptions révolutionnaires" ${ }^{12}$. Le souvenir des journées ouvrières d'avril et de juin 1848 reste trop présent pour que puisse se concevoir un déroulement harmonieux des opérations électorales. L'étendue des dispositions pénales inscrites dans le titre IV du décret organique du 2 février 1852 manifeste donc à sa manière une volonté de contenir la ferveur populaire.

10 C'est dans ce cadre que s'est déroulée la première expérience française du "vote universel". Un cadre qui n'allait plus guère changer dans la seconde moitié du siècle puisque l'édifice juridique hérité de l'épisode de 1848 allait garder ses grandes lignes jusqu'en 1913. En prohibant le recours à la violence, la législation était jugée suffisante pour faciliter la naturalisation d'un acte dont l'expérience de 1848 avait pourtant souligné l'imparfaite institutionnalisation. Sans perdre de vue ce caractère précaire, on peut tenter de résumer les lignes directrices du processus d'inscription spatiale et temporelle de l'acte du vote. Le lieu d'exercice de la civilité électorale peut-être analysé à partir de quatre ambitions normatives :

11 L'espace public du vote doit être reconnu par les électeurs comme un lieu pacifié, préservant des pressions et des violences extérieures. C'est un domaine marqué par des frontières tangibles et protectrices; ce qui autorise le libre déroulement de l'activité civique des acteurs sociaux. En tant que lieu public, le bureau de vote est soumis à un 
contrôle strict qui l'autonomise du reste du territoire de la commune. Il se différencie, par là, d'un dehors immédiat dont la surveillance s'avère beaucoup plus relâchée.

C'est un espace réglementé où la présence des agents de la force publique est fortement limitée. Celle-ci échappe à l'autorité étatique pour n'appartenir qu'à l'appréciation du président de bureau car c'est lui et lui seul qui est en mesure d'appeler des agents armés dans la salle du scrutin pour y maintenir l'ordre. L'autonomie de décision consentie aux assemblées électorales n'est cependant pas sans contrepartie. Dans la pratique, les mesures de police prises par le président de bureau sont étroitement surveillées afin d'éviter leur détournement à des fins partisanes.

13 C'est un espace consacré : l'inscription spatiale et temporelle du vote obéit à une triple règle de visibilité, de familiarité et de permanence. Le lieu (salle d'école, mairie, halle de place publique) comme les horaires du scrutin doivent dans cette optique bénéficier d'un degré d'évidence suffisant pour ne pas dérouter l'électeur. La tenue de l'assemblée électorale dans un lieu connu et solennel, le respect des horaires d'ouverture et de fermeture : autant d'éléments que la jurisprudence électorale viendra rapidement codifier avec précision.

14 C'est enfin un espace neutralisé car soustrait aux bruits et rumeurs de l'agitation politique et affecté au seul "devoir civique". L'accomplissement du geste électoral est conçu comme réclamant une ambiance calme et silencieuse qu'aucune violence, qu'aucun discours, qu'aucune rumeur ne peut contrarier. Il s'agit d'offrir à l'électeur toute la sérénité que l'importance de son acte suppose. C'est pourquoi, avant même l'installation des isoloirs, la salle de vote participe largement de l'isolement du vote.

Le suffrage universel comme arme de l'électeur

Les modalités juridiques mises en place tout au long du XIXème siècle pour pacifier l'espace électoral ne sauraient à elles seules rendre compte du rapport complexe qu'entretient le droit de suffrage avec la violence. En faisant du bureau de vote un conservatoire des vertus civiques, les partisans du "vote universel" espèrent modifier profondément les pratiques politiques en vigueur. Avec l'affirmation de normes comme l'individualisme électoral ou l'égalité numérique des voix s'impose une métamorphose des formes d'expression traditionnelles. Les plus violentes sont considérées désormais comme illégitimes parce que contraires à l'idée d'ordre social et politique. Louis Blanc, ancien quarante-huitard, se fait en 1874 l'avocat d'une telle évolution devant la représentation nationale: "Dire que le suffrage universel transforme les gouvernants en mandataires du peuple et les force comme tels à servir les besoins et à s'associer aux idées que le mouvement graduel de la civilisation apporte aux sociétés, c'est assez dire que le suffrage universel est un instrument de progrès ; mais il est aussi un instrument d'ordre. Je me trompe, il est l'instrument d'ordre par excellence. (Bravos à gauche). Et pourquoi ? Parce qu'en faisant de la loi l'oeuvre de tous, il l'impose au respect de tous... (nouveaux bravos à gauche); parce qu'en permettant à chacun de poursuivre sans violence le redressement de ses griefs, il désarme la violence... (très bien! très bien ! à gauche) ; parce qu'il investit le pouvoir émané de lui d'une force morale immense et qui rend toute entreprise factieuse impossible.(...) Je répète: parce qu'il rend toute entreprise factieuse impossible et dispense de recourir à l'emploi de la force matérielle"13.

16 Au delà de sa gravité, le vocabulaire employé par l'ancien rédacteur de la Revue du Progrès témoigne de l'ambition conservatrice du suffrage. En devenant universel, l'acte du vote débouche sur un ordre conventionnel qui "désarme la violence". Il vient 
interdire l'usage d'un certain répertoire d'action politique. En se voyant chargé d'exprimer fidèlement la souveraineté du peuple, le vote acquiert les attributs symboliques du sacré. La mise en forme spatiale du vote aboutit à un ensemble d'interdits qui protège et isole le dépôt du bulletin dans l'urne. Elle établit, ce faisant, une "sorte de vide logique" ${ }^{14}$ qui rend coûteuse toute entrée en contact avec l'espace électoral. De fait, l'apprentissage du bureau de vote est considéré comme une opération délicate appelant une éducation civique préalable ; éducation grâce à laquelle l'électeur doit non seulement se familiariser avec l'environnement électoral mais également intérioriser la solennité de l'acte à accomplir. Il s'agit, comme le proclamera Paul Bert, d'"inspirer à l'enfant un respect quasi-religieux pour ce grand acte de vote qui, jusqu'à présent, est par tant de personnes encore traité si légèrement..." ${ }^{15}$. L'accoutumance électorale transite par l'adoption de représentations qui vont progressivement renforcer la dimension sacrée du bureau de vote. La contribution de ces schémas d'action au processus de pacification doit être soulignée. On oublie trop que l'avènement du suffrage universel n'abolit pas automatiquement et immédiatement les formes violentes d'expression identitaire ${ }^{16}$. L'apprentissage de la technologie du vote se double d'une entreprise d'acculturation qui vise à récuser certaines formes populaires du politique issues d'un passé sans âge. Aussi, pour que l'idéal républicain s'inscrive dans les pratiques quotidiennes et investisse les croyances du plus grand nombre, il fallait que les élites urbaines forgent et surtout diffusent de nouvelles modalités de délégation électorale. L'école publique jouera ici un rôle primordial ${ }^{17}$. En dénonçant certaines manifestations de violence comme des stigmates d'une mentalité "archaïque" ou "primitive", les manuels scolaires vont contribuer à faire du vote la seule expression politique compatible avec l'exigence de dignité citoyenne. L'entreprise de rationalisation des comportements ainsi engagée apparait donc comme le prolongement inévitable de l'impératif politique d'échapper à la violence.

Le souci de promouvoir une expression électorale sincère et rationnelle se confond dans la littérature scolaire avec la volonté d'inculquer au futur citoyen une image valorisante de ses devoirs d'électeur. Cet apprentissage vise in fine à modifier le comportement des enfants et de leurs parents afin de les sensibiliser à la gravité d'une telle procédure. Se donnant pour objectif de transmettre un nouveau savoir civique et, par là même, de procéder à une inculcation morale explicite, les manuels d'instruction soulignent le changement profond qu'instaure l'avènement du suffrage universel. Si cet événement s'inscrit, selon leurs auteurs, dans la continuité de la Grande Révolution, la référence à 1789 ne manque pas d'ambiguïté. De leur point de vue, l'importance décisive de la Révolution mérite d'être saluée, mais à une condition : que le triomphe de l'idéal démocratique disqualifie dans le même temps et à jamais tout projet révolutionnaire. Le fait que les idées et les espoirs de 1789 aient été concrétisés par l'avènement de la République discrédite le recours à une révolution devenue inutile et dangereuse. Dès lors, il n'est pas inexact de dire que, pour eux, la Révolution est terminée : la commémoration dont elle fait l'objet ne fait plus que traduire son éviction du répertoire de la participation citoyenne.

La participation politique attendue du citoyen se résume donc à une activité spécifique : celle par laquelle les membres de la Nation prennent collectivement part à la sélection des dirigeants et participent indirectement à la formation des politiques publiques. Le vote devient, ce faisant, la forme légitime sinon exclusive de la participation citoyenne. Les républicains n'ont de cesse de le présenter comme un acte pacifique, organisé, régulier et prévisible; un acte en tout point opposé aux formes 
"primitives" de participation politique définies elles comme violentes, inorganisées et irrégulières. Ce renversement a une valeur symbolique exemplaire qu'illustre la substitution imaginée entre le fusil et le bulletin de vote. Chez Jules Steeg, la démonstration s'organise au travers de ce couple célèbre: "Dans la République, le gouvernement est confié pour un temps à des hommes élus par leurs concitoyens et responsables de leurs actes. Si le pays est content d'eux, ils les maintient au pouvoir; s'il n'en est pas content, il les change, sans bruit, sans violence, par le seul effet de ses suffrages. Dans une monarchie, on n'arrive à se débarrasser d'un maitre, oppresseur ou injuste, qu'au moyen d'une révolution; le fusil est l'instrument de la délivrance. Dans une république, l'arme toute puissante au moyen de laquelle on arrive à conquérir toutes les libertés et réaliser tous les progrès, c'est le bulletin de vote"18.

19 Complément de la monopolisation étatique de la coercition légitime, la représentation dominante du vote en fait le seul mode autorisé de gestion des conflits. En stigmatisant une violence devenue illégitime, les manuels diffusent ce que l'on pourrait appeler l'idéologie du consensus majoritaire: le suffrage universel et, en particulier, la règle majoritaire, fondent seuls désormais la légitimité des gouvernants. La possibilité d'une alternance régulière est présentée comme devant inciter les citoyens à la patience. Investi de toutes les espérances, le bulletin de vote devient "l'arme" par excellence du changement social.

De la politisation à l'acculturation

La réussite de l'entreprise d'acculturation inscrite dans l'apprentissage du vote passe, plus généralement, par un changement profond de "l'économie psychique" des individus (Norbert Elias). De par son existence, l'acte du vote renforce l'obligation pour l'individu de donner à son activité une orientation qui tienne compte deson insertion dans la société citoyenne, c'est-à-dire des liens d'interdépendance qui le rattachent à ses concitoyens. La salle de vote peut-être considérée, en ce sens, comme une sorte de laboratoire civique qui impose aux électeurs un renforcement de l'auto-contrainte de la vie pulsionnelle et affective. L'espace électoral est conçu comme le point de départ d'un procès de diffusion des différents comportements définissant le "bon citoyen".

21 Réunis dans un même lieu pour participer ensemble au choix des gouvernants, les individus sont tenus de se plier à un rôle - celui du citoyen-électeur - et donc de se conformer à des obligations implacables ${ }^{19}$. L'interdépendance ainsi que la forclusion de la violence imposée au citoyen passent par la reconnaissance pleine et entière des devoirs liés à l'appartenance au corps électoral. En instaurant une forme inédite de coprésence civique - ce que symbolise la fusion des bulletins dans une même urne de même que le résultat qui les agrége -, le vote préfigure l'harmonie qui doit régner dans une Nation dont la souveraineté a été définitivement restaurée. L'intérêt de cette formalisation est d'appeler l'attention sur la contribution du vote à l'intégration nationale via l'élaboration d'un ethos adapté aux règles du jeu démocratique. Dans ce cadre, la politisation renvoie au développement d'un "réflexe électoral" ${ }^{20}$ ou d'"une attention accordée au déroulement de la compétition politique" ${ }^{21}$, mais sans doute aussi à une capacité d'auto-contrainte. La réussite de cette entreprise de politisation repose sur l'aptitude à s'astreindre volontairement aux types de comportement imposés par le mode de division du travail politique que définit la citoyenneté républicaine. Il s'agit d'habituer les votants à une maîtrise de leurs attitudes corporelles et affectives, à une régulation réflexive de leurs actes. C'est dire que la mobilisation politique prend place 
dans une entreprise plus générale d'acculturation menée, de façon concomitante, dans toutes les sphères de la vie sociale.

De façon ultime, ce projet d'acculturation républicaine vise à démontrer que le citoyen ne serait rien en dehors de la société. Que son sort, sa voix, sa vie, dépendent d'une volonté politique exogène: celle qu'élabore la masse anonyme des autres électeurs. Sans faire de la société un système de rapports nécessaires ou un ordre prédéterminé, les républicains défendent l'idée d'une solidarité rationnellement acceptée car avantageuse pour tous. Cette interdépendance doit déboucher sur un monde débarrassé des menaces de désagrégation sociale. La nature du geste électoral est donc double: le vote est certes un acte individuel, manifestant la rationalité d'un choix élaboré en conscience; toutefois, il manifeste aussi l'appartenance à un groupe, la totalité souveraine de la nation, dont l'existence prohibe l'usage de la violence. L'isolement dont bénéficie le geste électoral, dans l'enceinte sacrée du bureau de vote, n'est que provisoire. A en croire les discours républicains, un tel dispositif préparerait l'avènement d'une ère nouvelle : celle qui verra la société environnante adopter les normes pacificatrices qu'établit la mise en forme spatiale du vote. Ce jour là, le principe de séparation entre l'espace civique et l'espace social sera aboli. Une même continuité reliera les comportements privés et publics : la communauté des individus ne fera plus qu'une avec la société des citoyens.

Lire la suite

\section{NOTES}

1. Weber (M.), Economie et société, Paris, Plon, 1971, p.57.

2. Ibid., p.58.

3. L'affirmation de Maurice Block en témoigne : "La violence est antipathique à la vertu civique. Le citoyen doit toujours employer des moyens légaux, et si les lois sont mauvaises, ce sont encore les voies légales qu'il faut suivre pour arriver à leur changement. Il doit savoir attendre et supporter avec dignité les inconvénients dont il se plaint, et lorsqu'il se sent le plus fort, c'est avec modération qu'il doit user de son pouvoir." ; "Vertus civiques" in Block (M.) dir., Dictionnaire général de la politique, Paris, O. Lorenz, nouvelle édition, 1873, p 1109.

4. Daumier (H.), "V'là ma cartouche", dessin paru dans le Charivari du 20 novembre 1869. Document reproduit dans Daumier (H.), Moeurs politiques, Catalogue et notices de Jacqueline Armingeat, Paris, Editions Michèle Trinckvel, 1982. Le maintien dans le discours des guesdistes de la fameuse formule "par le bulletin ou par le fusil" montre cependant que cette pacification sociale ne touche pas de façon identique tous les acteurs politiques.

5. Cf. notamment sur ce point, Rosanvallon (P.), Le sacre du citoyen. Histoire du suffrage universel, Paris, Gallimard, 1992, p.290.

6. Cité par Kéguelin de Rozières (G.), Crimes et délits électoraux, Thèse pour le doctorat de droit, Paris, A. Rousseau, 1904, p.37 et sq., dont ces développements sont inspirés. 
7. Anthony Giddens attire l'attention sur la nécessité pour le sociologue de "tenir compte du caractère "situé" de l'interaction dans le temps et dans l'espace" in La constitution de la société, Eléments de la théorie de la structuration, Paris, P.U.F., 1987, p.163. A cet effet, il l'invite à s'intéresser aux modalités de construction des espaces de rencontre entre les acteurs sociaux. L'intérêt de la théorie de la structuration proposée par A. Giddens réside en bonne partie dans ce souci de tenir compte de l'organisation temporelle et spatiale des activités sociales. L'institutionnalisation de la pratique électorale renvoie, à l'origine, à l'inscription dans le temps et dans l'espace de l'acte de vote. En temps qu'"occasion sociale", l'expression est empruntée à Erving Goffman, le vote rassemble, en effet, en un même lieu un grand nombre d'acteurs sociaux. Ce lieu structure le comportement des électeurs et les amène à intérioriser progressivement un certain nombre de valeurs et de normes d'attitude.

8. Cf. Elias (N.), La civilisation des moeurs, Paris, Calmann-Lévy, 1973, (1ère éd. 1939) et La dynamique de l'Occident, Paris, Calmann-Lévy, 1975, (1ère éd. 1939). Sur ce point, la contribution de Claudine Haroche.

9. Sur ce dispositif réglementaire et ses présupposés politiques, cf. Ihl (0.), La genèse empirique d'une abstraction. Sur la naissance de l'urne électorale, Rapport au Colloque L'acte de vote en question. Expériences françaises et étrangères de la pratique électorale, organisé par le C.R.P.S. et l'A.F.S.P., Paris, Centre Malher, 8-9 décembre 1992. 10. Sur ce dispositif réglementaire et ses présupposés politiques, cf. Ihl (0.), La genèse empirique d'une abstraction. Sur la naissance de l'urne électorale, Rapport au Colloque L'acte de vote en question. Expériences françaises et étrangères de la pratique électorale, organisé par le C.R.P.S. et l'A.F.S.P., Paris, Centre Malher, 8-9 décembre 1992. 11. Autrement dit, un ensemble de transactions et de contraintes devenues "invisibles" à force d'être routinières. Douglas (M.), How Institutions Think, New York, Syracuse University Press, 1986.

12. Extrait d'un article paru dans l'Assemblée nationale du 19 septembre 1848, cité par Balland (R.), "De l'organisation à la restriction du suffrage universel en France (1848-1850)" in Réaction et suffrage universel en France et en Allemagne, Paris, 1963, p. 81.

13. Blanc (L.), "Le suffrage universel (Assemblée nationale, session du 4 juin 1874 ", in Discours politiques (1847-1881), Paris, Librairie Germer-Baillière et Cie, 1882, p.182. 14. Durkheim (E.), Les formes élémentaire de la vie religieuse, Paris, P.U.F., Coll. Quadrige, 1985, (1ère éd. 1912), p.55.

15. Bert (P.), De l'éducation civique, Conférence faite au palais du Trocadéro le 6 août 1882 au profit des bibliothèques populaires syndiquées du département de la Seine, Paris, Picard \& Bernheim, s.d., p.15.

16. Cf. Corbin (A.), "L'histoire de la violence dans les campagnes françaises au XIXe siècle. Esquisse d'un bilan", Ethnologie française, vol.XXI, n³, 1991, pp.224-235.

17. Cf. Déloye (Y.), La citoyenneté au miroir de l'école républicaine et de ses contestations : Religion et politique en France XIXe-XXe siècle, Thèse pour le doctorat en science politique Université Paris I, 1991, multigra.

18. Steeg (J.), Instruction morale et civique, Paris, Librairie classique N. Fauvé et $\mathrm{F}$. Nathan, 1882, pp.134-135.

19. Comme le montre l'étude des bulletins annulés, certains électeurs résisteront à cette obligation. Ils utiliseront leur bulletin de vote dans le sens d'une prise de parole bruyante et contestataire. Voir sur ce point, Déloye (Y.), Ihl (O.), "Légitimité et 
déviance. L'annulation des votes dans les campagnes de la IIIe République", Politix, $\mathrm{n}^{\circ} 15$, troisième trimestre 1991, p.20 sq.

20. L'expression est empruntée à Offerlé (M.), "Mobilisation électorale et invention du citoyen : l'exemple du milieu urbain français à la fin du XIXème siècle", dans Gaxie (D.) dir., Explication du vote, Un bilan des études électorales en France, Paris, Presses de la F.N.S.P., 1985, p.155.

21. Gaxie (D.), Le cens caché, Paris, Le Seuil, 1978, p.46.

INDEX

Index géographique : France

Mots-clés : histoire, sociologie historique, démocratie 